\title{
PDX-1 haploinsufficiency limits the compensatory islet hyperplasia that occurs in response to insulin resistance
}

\author{
Rohit N. Kulkarni, ${ }^{1}$ Ulupi S. Jhala, ${ }^{2}$ Jonathon N. Winnay, ${ }^{1}$ Stan Krajewski, ${ }^{3}$ \\ Marc Montminy, ${ }^{4}$ and C. Ronald Kahn'

\begin{abstract}
${ }^{1}$ Research Division, Joslin Diabetes Center and Department of Medicine, Harvard Medical School, Boston, Massachusetts, USA.
\end{abstract} \\ ${ }^{2}$ The Whittier Institute, University of California, San Diego, La Jolla, California, USA. ${ }^{3 T h e ~ B u r n h a m ~ I n s t i t u t e, ~ L a ~ J o l l a, ~ C a l i f o r n i a, ~ U S A . ~}$ \\ ${ }^{4}$ Peptide Biology Laboratories, The Salk Institute for Biological Studies, San Diego, California, USA.
}

\begin{abstract}
Inadequate compensatory $\beta$ cell hyperplasia in insulin-resistant states triggers the development of overt diabetes. The mechanisms that underlie this crucial adaptive response are not fully defined. Here we show that the compensatory islet-growth response to insulin resistance in 2 models - insulin receptor (IR)/IR substrate-1 (IRS-1) double heterozygous mice and liver-specific IR KO (LIRKO) mice - is severely restricted by PDX-1 heterozygosity. Six-month-old IR/IRS-1 and LIRKO mice both showed up to a 10-fold increase in $\beta$ cell mass, which involved epithelial-to-mesenchymal transition. In both models, superimposition of PDX-1 haploinsufficiency upon the background of insulin resistance completely abrogated the adaptive islet hyperplastic response, and instead the $\beta$ cells showed apoptosis resulting in premature death of the mice. This study shows that, in postdevelopmental states of $\beta$ cell growth, $P D X-1$ is a critical regulator of $\beta$ cell replication and is required for the compensatory response to insulin resistance.
\end{abstract}

\section{Introduction}

Islet hyperplasia and hyperinsulinemia develop to varying degrees in virtually all states of insulin resistance and are apparent in humans, rodents, and other mammals in the presence of obesity, genetic insulin resistance, and states of stress or when counterinsulin hormones are chronically elevated (reviewed in ref. 1). The factors that stimulate growth, the specific proteins in the $\beta$ cells, and the precise mechanisms that regulate this compensatory hyperplasia in insulin resistance remain poorly defined (reviewed in ref. 1). The pancreatic homeodomain protein PDX-1 and the insulin/IGF-I signaling pathway are both important for growth and cell proliferation in the pancreas. While PDX-1 has been shown to regulate expansion of pancreatic progenitor cells (reviewed in ref. 2), proteins in the insulin/IGF-I signaling pathway are known to regulate growth, cell proliferation, adhesion, and tissue architecture as well as to modulate metabolism in virtually all tissues in mammals, including the pancreatic islets $(3,4)$.

PDX-1 regulates target gene transcription both as a monomer and as a heterodimer with the three amino acid loop extension homeodomain protein PBX-1. The monomeric and dimeric forms of PDX regulate specific and distinct targets. PDX-PBX dimerization has been shown to be critical for embryonic pancreatic cell proliferation (5-7). PBX-1-null mice display an approximately $30 \%$ decrease in pancreatic cell proliferation, while mice expressing the PBX-1 interaction-deficient PDX-1, on a PDX-null background, manifest a severe attenuation of pancreatic cell expansion during embryogenesis (7). Mice with PDX-1 heterozygosity have been reported to exhibit enhanced $\beta$ cell

Nonstandard abbreviations used: IR, insulin receptor; IRS, IR substrate; LIRKO, liver-specific IR KO; PCNA, proliferating cellular nuclear antigen; TKO, IR/IRS-1/ PDX-1 triple heterozygous KO.

Conflict of interest: The authors have declared that no conflict of interest exists.

Citation for this article: J. Clin. Invest. 114:828-836 (2004)

doi:10.1172/JCI200421845 apoptosis (8). On the other hand, mice with a $\beta$ cell-specific $\mathrm{KO}$ of the insulin receptor (IR) exhibit decreased islet growth in adults and a susceptibility to developing overt diabetes $(9,10)$. Although mice with IR substrate-1 (IRS-1) deficiency show attenuated nutrient sensing in the islets, the compensatory islet hyperplasia in response to insulin resistance is maintained $(11,12)$. Similarly, mice double heterozygous for the IR and IRS-1 (also called IR/IRS-1 mice) and liver-specific IR KO (LIRKO) mice both have severe insulin resistance that results in massive compensatory hyperplasia with up to a 10 -fold increase in $\beta$ cell mass $(13,14)$. By contrast, deficiency of IRS- 2 results in hyperglycemia, $\beta$ cell apoptosis $(12,15,16)$, and a strain-dependent dysregulation of PDX-1 expression (17). To explore the direct link between PDX-1 and the insulin resistance-dependent proliferative response of $\beta$ cells, we crossed IR/IRS-1s or LIRKOs with PDX-1 heterozygous mice (PDX-1 mice). Our data demonstrate a crucial role of PDX-1 as a regulator of growth and proliferation of adult $\beta$ cells in insulin-resistant states and complement the recent observation that $\beta$ cell replication is important in the maintenance of $\beta$ cell mass (18).

\section{Results}

Growth and development of IR/IRS-1/PDX-1 triple heterozygous $K O$ mice. All mice were born in a normal mendelian ratio in the different groups, and no embryonic lethality was observed. Mice that were heterozygous for IRS- 1 - the IRS- 1 heterozygous (IRS-1), IR/IRS-1 double heterozygous (IR/IRS-1), IRS-1/PDX-1 double heterozygous (IRS-1/PDX-1), and IR/IRS-1/PDX-1 triple heterozygous KO (TKO) groups - exhibited a significant (10-15\%) reduction in body weight (Figure $1 \mathrm{~A}$, left and right panels), consistent with earlier reports $(11,19)$. The further decrease in body weights in TKO and IRS-1/PDX-1 males and females compared with the IRS- 1 and IR/IRS- 1 groups is likely caused by glycosuria and wasting due to severe diabetes in older mice. Qualitatively similar but milder phenotypes were observed in females. Therefore, data from males only will be presented. 
A

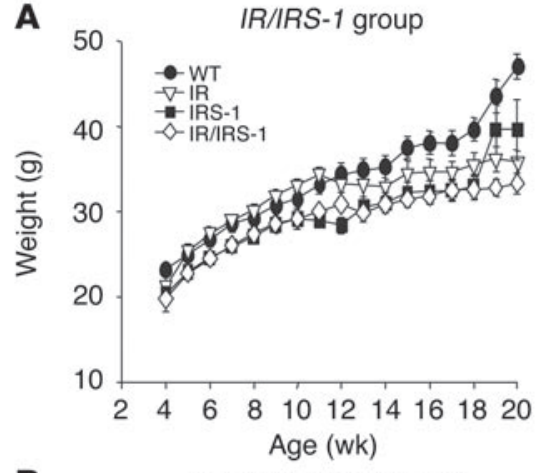

B
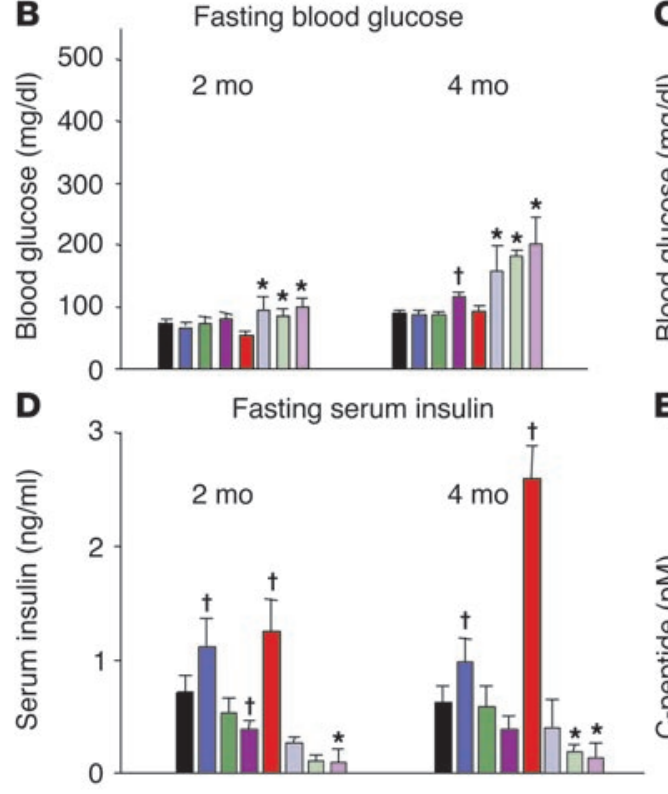

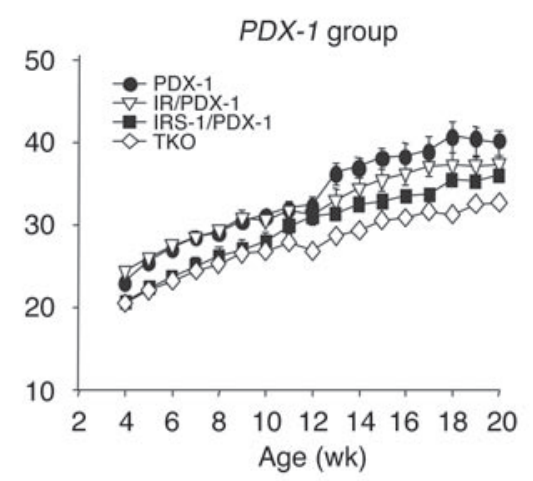

C

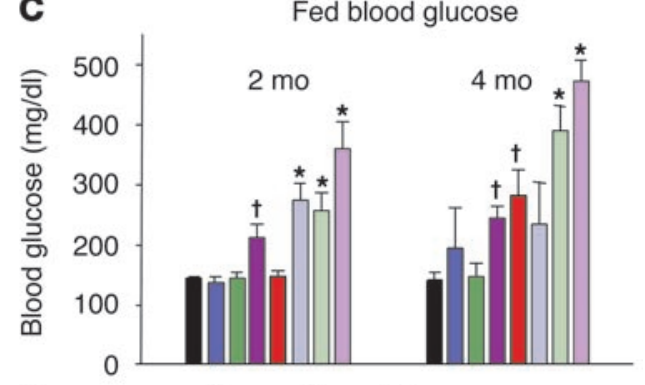

E

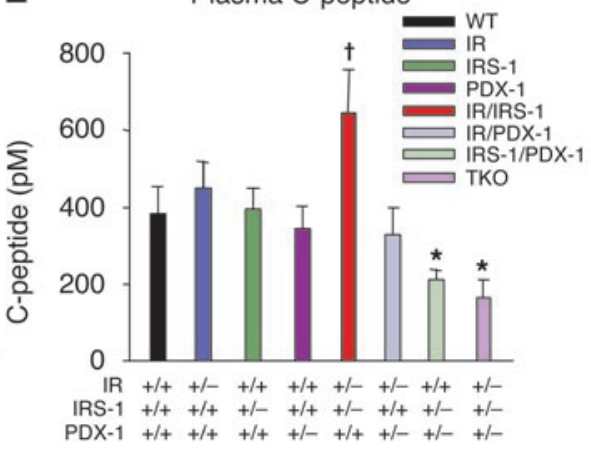

Figure 1

Growth curves and alterations in blood glucose and serum insulin levels. (A) Body weights of male mice were determined between the ages of 4 and 20 weeks. Mice are divided into IR/IRS-1 (left panel) and PDX-1 (right panel) groups. $P<0.05$, IRS- 1 or IR/IRS- 1 vs. WT at all time points; $P<0.05$, IR vs. WT from 12 weeks onward; $P<0.05$, IRS-1/PDX- 1 or TKO vs. PDX-1 at all time points, IR/ PDX-1 vs. PDX-1 from 12 weeks onward ( $n=12-26)$. (B) Fasting blood glucose was measured after a 14-hour overnight fast in 2- and 4-month-old male mice. (C) Fed blood glucose was measured in random-fed 2- and 4-month-old male mice. (D) Fasting serum insulin was measured after a 14-hour overnight fast in 2- and 4-monthold male mice. (E) C-peptide levels were measured by RIA. ${ }^{*} P<0.05$, IR/PDX-1, IRS-1/PDX-1, or TKO vs. PDX-1; ${ }^{\dagger} P<0.05, \mathrm{IR}, \mathrm{IR} / \mathrm{IRS}-1$, or PDX-1 vs. WT ( $n=10-22)$.
TKO mice manifest severe byperglycemia, bypoinsulinemia, loss of acute phase insulin secretion, and glucose intolerance. IR/IRS-1 mice showed normal glucose levels at 2 months that worsened slightly at 4 months of age, especially in the fed states. In PDX-1 heterozygotes, however, the fed blood glucose was mildly elevated at 2 months, and the hyperglycemia persisted at 4 months. At this time IR and IR/IRS-1 mice showed hyperinsulinemia (13), and this was adequate to maintain normoglycemia. By age 4 months, the double heterozygous mice showed hyperglycemia despite the presence of even higher levels of circulating insulin (Figure 1, B-D). In mice with combined heterozygosity for PDX-1, IR, and/or IRS-1, we detected significantly higher blood glucose levels in the fasted and fed states by 2 months, and the hyperglycemia worsened by 4 months in all groups, except IR/ PDX-1 compared with PDX-1 single heterozygotes (Figure 1, B and C). The hyperglycemia in IRS-1/PDX-1 and TKO compound KOs could be attributed to significantly lower serum insulin levels compared with those in individual heterozygotes and controls (Figure 1D). Plasma C-peptide, measured at 3 months of age, showed a similar trend, suggesting a significantly reduced insulin output from the endocrine pancreas (Figure 1E).

Insulin/IGF-I signaling in $\beta$ cells has been demonstrated to play a role in glucose-stimulated insulin secretion (20-22), while PDX-1 is known to regulate expression of Glut 2 and glucokinase genes $(23,24)$. Furthermore, insulin/IGF-I signaling in the islet has been linked with glucokinase $(10,21,25,26)$ and PDX-1 expression (26). To evaluate insulin secretory function, mice were injected with glucose by the intraperitoneal route. An approximately 3-fold secretory response was observed in the WT, while virtually no responses were observed in all the groups that were heterozygous for PDX-1, consistent with an earlier study (Figure 2, A and B) (27). Consequently, the groups heterozygous for PDX-1 displayed glucose intolerance by 2 months, and the glucose levels were highest at all time points during the glucose tolerance test in IRS-1/PDX-1 and TKO mice (Figure 2D), compared with respective controls (Figure 2C), and continued to worsen as the mice aged (Figure 2, $\mathrm{E}$ and $\mathrm{F}$ ). We observed mild glucose intolerance in the PDX-1 group (Figure 2D), while the IR/IRS-1 mice were glucose intolerant only at age 4 months (Figure 2E). We did not observe significant differences in insulin sensitivity, as measured by an insulin tolerance test, between the groups at 2 months (data not shown). The severe hyperglycemia in the IRS-1/PDX-1 and TKO groups is likely due to relatively low circulating insulin levels in the compound KOs.

Altered PDX-1 protein expression and reduced $\beta$ cell mass in TKO islets. Western blotting of proteins from whole pancreas, to quantify the partial loss of PDX-1, showed approximately $50 \%$ lower 

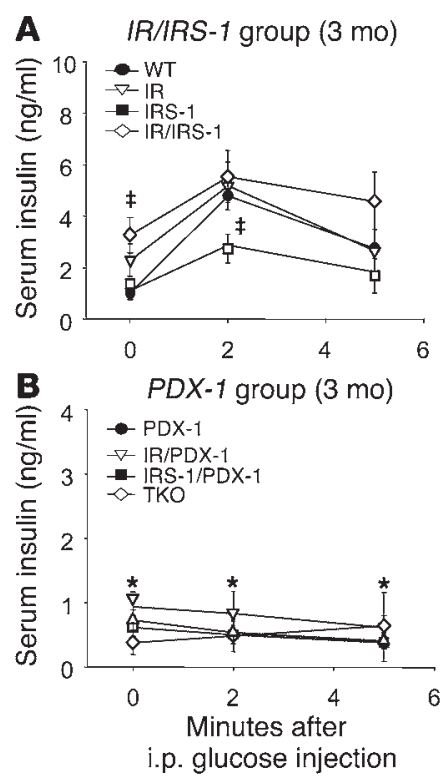

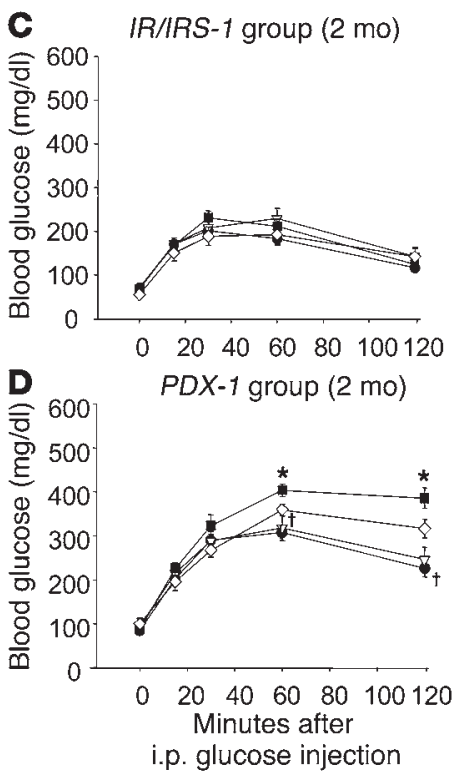

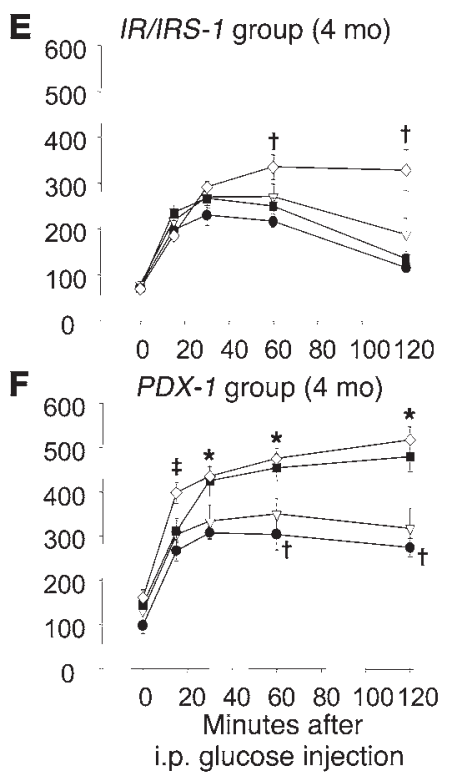

Figure 2

Alterations in acute phase response to glucose and glucose tolerance. (A and $\mathbf{B})$ Acute phase insulin secretory response to intraperitoneal (i.p.) injection of glucose was measured in mice after a 14 -hour overnight fast. ${ }^{*} P<0.05$, WT vs. PDX-1, IR/PDX-1, IRS-1/PDX-1, or TKO; $¥ P<0.05$, IRS-1 or IR/IRS-1 vs. WT $(n=4-10)$. (C-F) Glucose tolerance after intraperitoneal injection of glucose was measured after a 14-hour fast in 2-month-old (C and $\mathbf{D})$ and 4-month-old (E and $\mathbf{F})$ male mice. In 2-month-old mice, ${ }^{*} P<0.05$, IRS-1/PDX-1 or TKO vs. PDX-1; ${ }^{\dagger} P<0.05$, PDX-1 vs. WT. In 4-month-old mice, ${ }^{*} P<0.05$, IRS-1/PDX-1 or TKO vs. WT; ${ }^{\dagger} P<0.05$, IR/IRS-1 or PDX-1 vs. WT $(n=7-18)$.

levels of PDX-1 protein in PDX-1 heterozygotes compared with the WT, IR, and IRS-1 groups (Figure 3, A and B). However, the PDX-1 levels were reduced even further in the IRS-1/PDX-1 and TKO groups compared with the PDX-1 group; in fact, PDX-1 was almost undetectable in these groups. By contrast, a 2.3-fold higher level of PDX-1 protein was observed in IR/IRS-1 mice compared with WT mice (Figure 3B). When PDX-1 was expressed per milligram islet protein, however, the values were not significantly different from those for the WT group, which suggests that the increase in PDX-1 protein levels in the pancreas in IR/IRS-1 mice is due to islet hyperplasia (Figure 3C).

Morphometric analysis of $\beta$ cell mass at 3 months of age showed a 2- to 4-fold increase in the IR/IRS-1 group (and about a 9-fold increase at 6 months; data not shown), compared with the WT (Figure 3, D and E) $(13,28)$. Similarly, IR and IRS-1 heterozygous mice showed a significant increase in $\beta$ cell mass, which indicates a compensatory response of islets to insulin resistance $(11,12,29)$. In contrast, we detected a milder, statistically insignificant reduction in $\beta$ cell mass in PDX-1 heterozygotes (30). When PDX-1 heterozygosity was combined with heterozygosity for 1 or more of the insulin signaling proteins (IR/PDX-1, IRS-1/PDX-1, and TKO), a dramatic decrease in $\beta$ cell mass was observed compared with that in PDX-1 heterozygotes alone (Figure 3E). In fact, we detected few or no islets in pancreas sections in a majority of IRS-1/PDX-1 and TKO mice. The reduced $\beta$ cell mass was consistent with reduced pancreatic insulin content in the various groups, the lowest content being observed in the IRS-1/PDX-1 and TKO groups (Figure 3F). We did not detect significant differences in non- $\beta$ cell mass among the groups (data not shown).

Decreased expression of PDX-1 targets in TKO mice. We evaluated pancreata from the various groups for expression of key markers of islet function including insulin (Figure 4A, upper panels) and glucagon (Figure 4A, green, middle panels). Insulin- and glucagon-positive cells could be detected in all genotypes, which indicates that differentiation of $\alpha$ and $\beta$ cells was not disrupted in the compound KOs. As expected, IR/IRS-1 mice showed an approximately 3 -fold increase in islet size, due to an increase in $\beta$ cell mass, with the non- $\beta$ cells scattered throughout the islets (Figure 4A, upper and middle panels) $(13,28)$. To examine whether the islet hyperplasia was due to an increase in non- $\beta$ cell types in the islets, we costained for expression of insulin, glucagon, and somatostatin in pancreas sections from IR/IRS-1 and PDX-1deficient groups (Figure 4B). The hyperplastic islets in IR/IRS-1 mice were characterized by an increase in $\beta$ cells with glucagonand somatostatin-positive cells scattered within the islet. Consistent with a lack of significant difference in circulating glucagon levels among groups, the number of glucagon- and somatostatinpositive cells appeared to be in similar proportions in all groups. Pancreas sections from IRS-1/PDX-1 and TKO mice showed very small islets with a reduced number of $\beta$ cells, which resulted in the appearance of an increase in glucagon-staining $\alpha$ cells within the core of the islet (Figure 4A, middle panels). The absence of a significant alteration in non- $\beta$ cell mass and in circulating glucagon levels (data not shown) in the compound KOs suggested a predominant effect on $\beta$ cells. Furthermore, we did not detect differences in circulating levels of total glucagon-like peptide-1 between groups, which suggests that the altered $\beta$ cell mass is unlikely to be linked with glucagon-like peptide- 1 action (WT, $14 \pm 4$; IR/IRS-1, $16 \pm 6$; PDX-1, $15 \pm 7$; IRS-1/PDX-1, $22 \pm 10$; TKO, $17 \pm 4 \mathrm{pmol} / \mathrm{l} ; n=3-5, P=\mathrm{NS})$.

We also examined islets for alterations in Glut2, a key target for PDX-1 (24). Robust Glut2 expression was detected in IR, IRS-1, and IR/IRS-1 double heterozygote islets (Figure 4A, lower panels). However, the protein was virtually undetectable in all mice carry- 


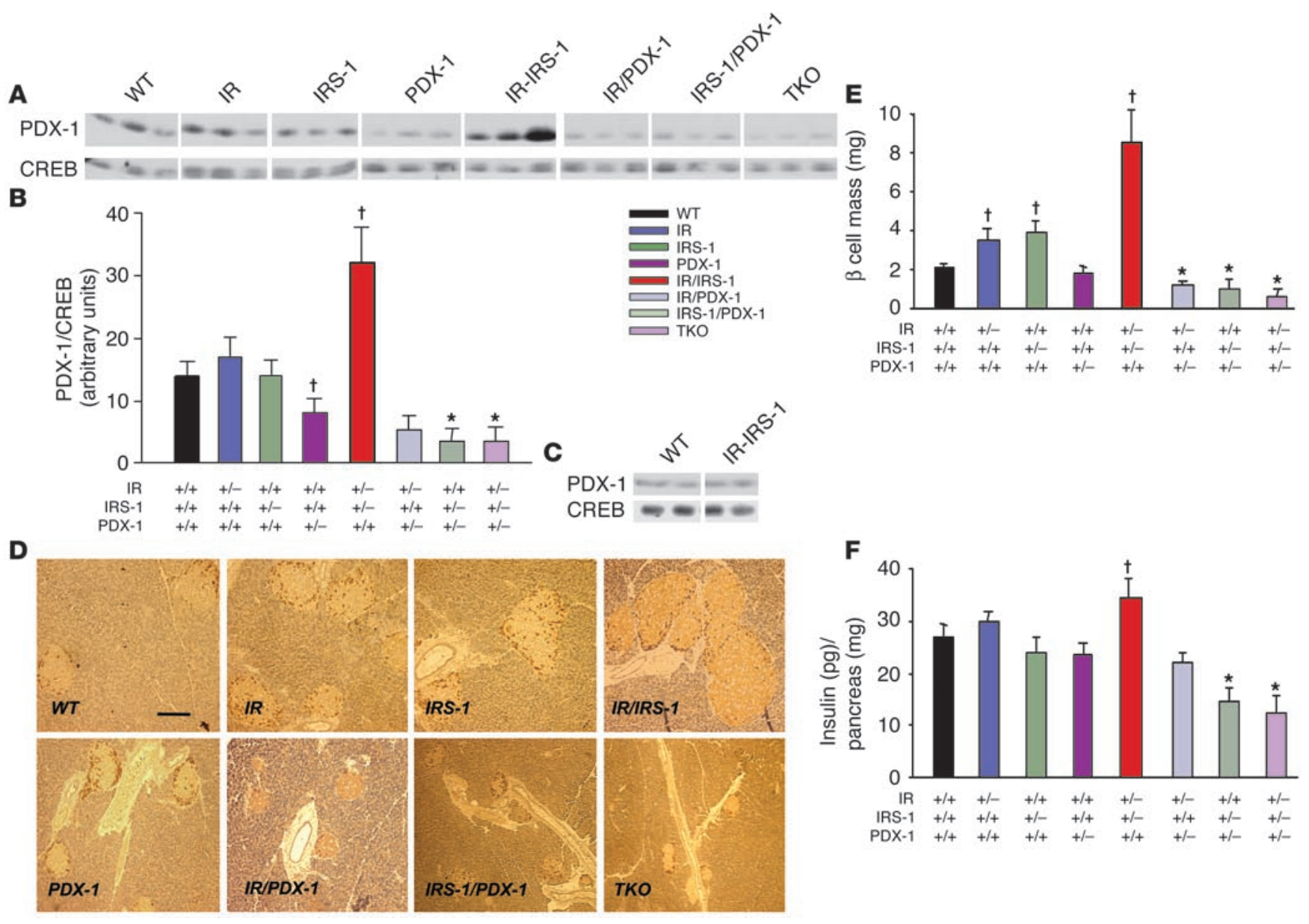

Figure 3

Expression of PDX-1 in pancreas and reduced $\beta$ cell mass and pancreatic insulin content in IRS-1/PDX-1 and TKO mice. (A) Western blotting of PDX-1 and CREB proteins prepared from pancreas as described in Methods. Each lane corresponds to an individual mouse ( $n=3$ ). (B) Quantitation of PDX-1 levels in A. Data are depicted as the ratio of PDX-1 to CREB and expressed as arbitrary densitometry units. ${ }^{*} P<0.05$, PDX-1 vs. IRS-1/ PDX-1 or TKO; ${ }^{\dagger} P<0.05$, PDX-1 or IR/IRS-1 vs. WT $(n=3)$. (C) Western blotting of PDX-1 and CREB proteins prepared from freshly isolated islets from WT and IR/IRS-1 mice $(n=2)$. (D) Representative pancreas sections were stained with cocktail antibody to non- $\beta$ cell hormones as described in Methods. Scale bar: $50 \mu \mathrm{m}$. (E) $\beta$ Cell mass was estimated by morphometric analysis as described in Methods. ${ }^{*} P<0.05$, IR/PDX-1, IRS-1/PDX-1, or TKO vs. PDX-1; $\uparrow<0.05$, IR, IRS-1, or IR/IRS-1 vs. WT. (F) Pancreatic insulin content was measured in acid-ethanol extracts of homogenized pancreas as described in Methods. ${ }^{*} P<0.05$, IRS-1/PDX-1 or TKO vs. PDX-1; ${ }^{\dagger} P<0.05$, IR/IRS-1 vs. WT $(n=4-6)$.

ing a single allele for PDX-1, including PDX-1, IRS-1/PDX-1, and TKO mice. These observations are consistent with earlier observations that Glut2 is a direct transcriptional target for PDX-1 (24). In addition, based on preliminary gene expression profiling studies in islets from PDX-1 heterozygotes (M. Montminy, U.S. Jhala, and J. Kushner, unpublished observations), we examined expression of synaptophysin, another PDX-1 target and an integral component of the neuroendocrine secretory granule (Figure $4 \mathrm{~A}$, red, middle panels). The expression pattern of synaptophysin paralleled that of Glut2 (Figure 4A, lower panels). Thus, in addition to regulating insulin, PDX-1 also regulates other genes that are important for glucose sensing and for insulin exocytosis, which may in part explain the loss of acute phase insulin secretion observed in the PDX-1-deficient mice.

Increased $\beta$ cell apoptosis and diminished $\beta$ cell replication in TKO islets. $\beta$ Cell mass in the adult mouse is maintained by a balance between newly generated $\beta$ cells (by $\beta$ cell replication and neogenesis from potential ductal precursors) and $\beta$ cell death by apoptosis or necrosis $(18,31)$. To obtain an estimate of these parameters and to isolate the potential mechanism underlying the poor islet compensation in the IRS-1/PDX-1 and TKO groups, we examined the pancreas for markers of proliferation and apoptosis in these mice $(32,33)$. In double heterozygous mice carrying both copies of PDX-1, we observed a striking pattern and increase in proliferating cellular nuclear antigen (PCNA) staining in cells within the islets, while virtually no $\mathrm{PCNA}^{+}$cells were evident in other groups (Figure 5A; Table 1). Furthermore, the $\mathrm{PCNA}^{+}$cells showed a distinct morphology compared with surrounding $\beta$ cells. In contrast, examination of the pancreas for activated caspase-3, a marker for the end stage of apoptosis, showed increased staining in islets of TKO mice. Some caspase- $3^{+}$cells were also observed in islets of IRS-1/PDX-1 mice, while few or none could be detected in other groups (Figure 5B; Table 1). Thus, the islet hyperplasia in IR/IRS-1 mice is predominantly caused by a robust expansion of $\beta$ cells, 
A

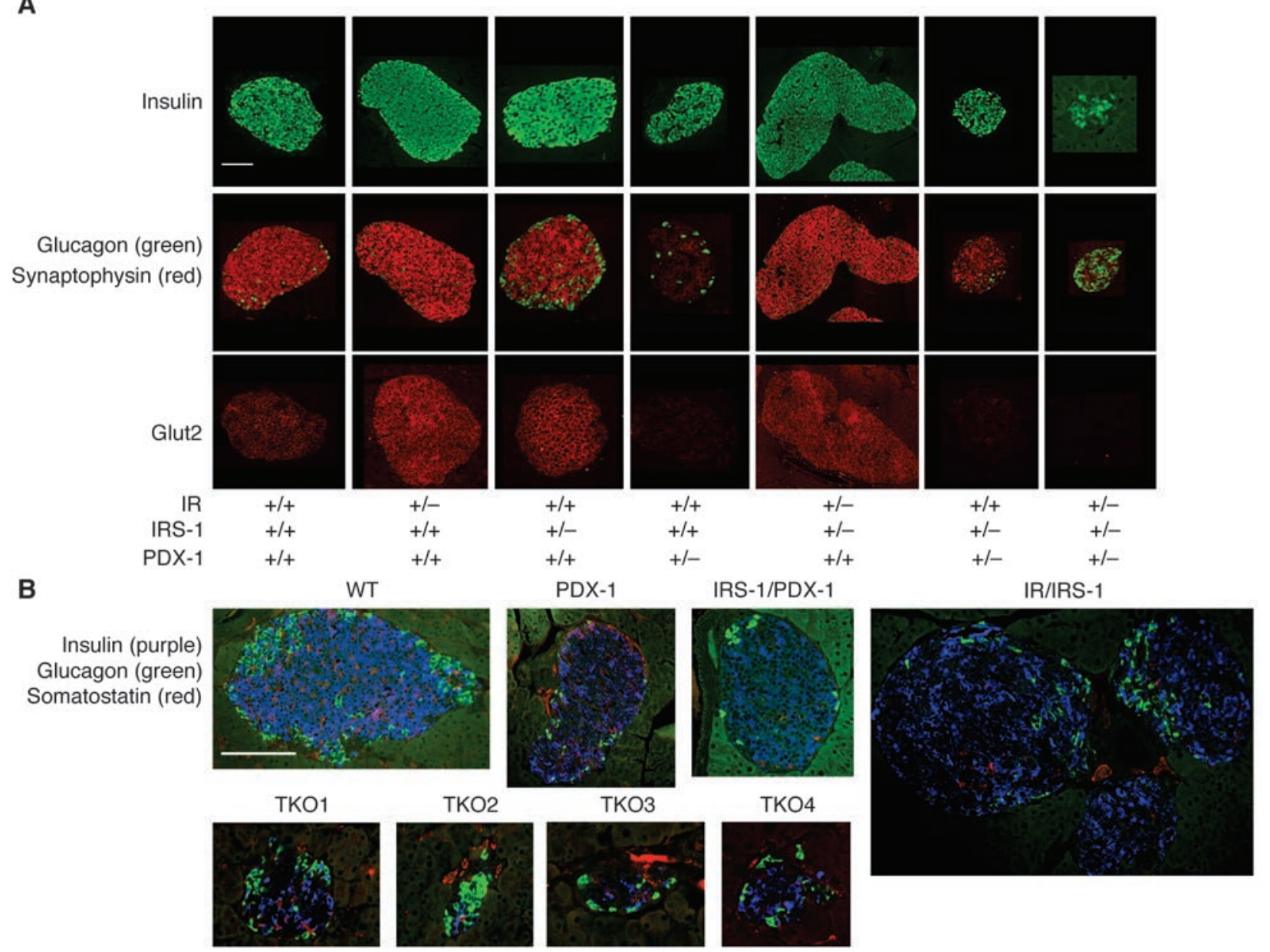

\section{Figure 4}

Immunostaining of pancreas sections for islet cell markers. (A) Representative islets from pancreas sections stained with immunofluorescent antibodies for insulin (upper panels, green), glucagon (middle panels, green), synaptophysin (middle panels, red), and Glut2 (lower panels, red) as described in Methods. (B) Representative islets from pancreas sections from the TKO group, costained for insulin (purple), glucagon (green), and somatostatin (red). Four different TKO islets are shown.

whereas the lack of compensatory response in IRS-1/PDX-1 and TKO groups is most likely a result of the combined absence of $\beta$ cell proliferation and increased $\beta$ cell apoptosis.

To evaluate the origin of the $\mathrm{PCNA}^{+}$cells, we stained serial sections of pancreas with a fluorescence-labeled duct-specific lectin (DBA) (34). Ductal epithelial cells showed the expected positive staining; however, none of the cells in the area of proliferation were positive for DBA-lectin immunohistochemistry for duct-specific glycoconjugates (Figure 5C). These data strongly suggest that the proliferating cells, rather than being of ductal origin, are likely replicating $\beta$ cells with metaplastic changes. Similar metaplastic changes in rapidly expanding epithelial cell types are associated with marked changes in adherens junctions between cells (4). In the pancreas, the cadherin-catenin complex regulates aggregation of $\beta$ cells in vivo (35) and participates in paracrine signal transmission from neighboring cells by interacting with growth factor receptors $(36,37)$. We therefore examined the islets for expression of 2 important components of the adherens junction, namely E-cadherin, a transmembrane protein that mediates cell-to-cell association, and $\beta$-catenin, which anchors E-cadherin to the cytoskeletal network in the cells $(4,35) . \beta$ Cells in WT islets stained positive for both E-cadherin and membrane-bound $\beta$-catenin, indicating strong cell-to-cell adhesion, which normally occurs in well-clustered islets (Figure 5D). E-cadherin, however, was dramatically downregulated and undetectable in the hyperplastic islets in IR/ IRS-1 mice (Figure 5D). In marked contrast, PDX-1 heterozygotes strongly expressed E-cadherin but showed a virtual absence of $\beta$-catenin, suggesting a fundamental alteration in adherens junctions in $\beta$ cells of the PDX- 1 islets (Figure 5D).

These data are reminiscent of changes in adhesion protein expression that are normally observed in rapidly proliferating cells, especially during tumorigenesis (4). In such a model, the loss of E-cadherin leads to a dissolution of the adherens junction and allows the membrane-bound $\beta$-catenin to migrate into the cytoplasm and nucleus and participate in activation of genes required for proliferation (4). Accordingly, the $\mathrm{PCNA}^{+}$cells in the hyperplastic islets from IR/IRS-1 mice showed a decrease in membrane-bound $\beta$-catenin and an increase in translocation into the cytoplasm and nucleus. In the surrounding nonproliferating $\beta$ cells, however, $\beta$-catenin was found to be membrane bound. The 
A
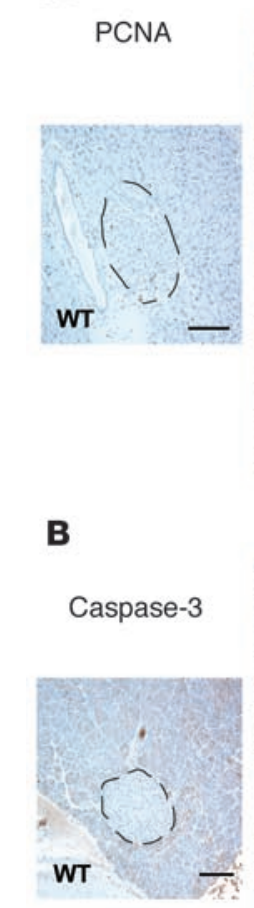

B
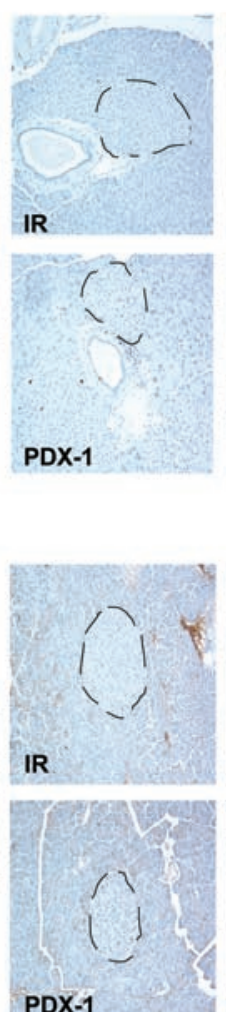

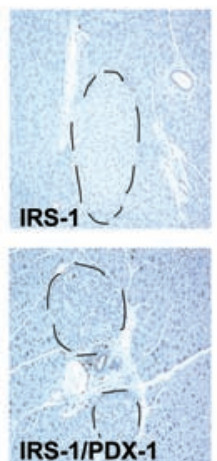

IRS-1/PDX-1

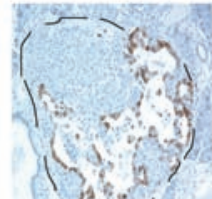

IR/IRS-1

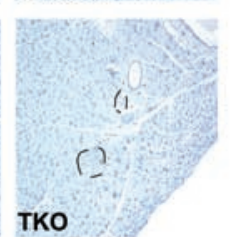

TKO
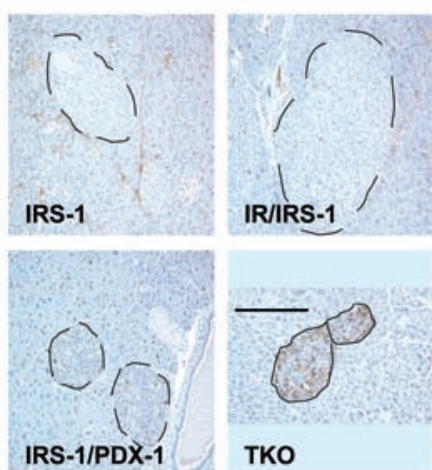

TKO
C
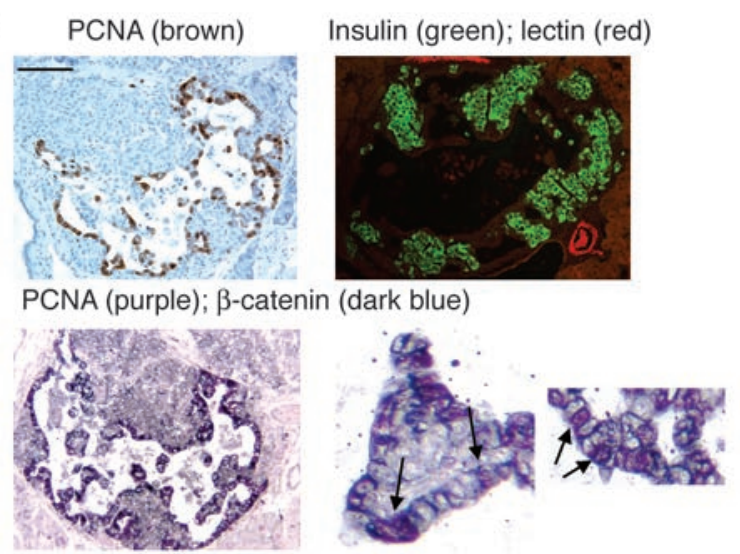

D
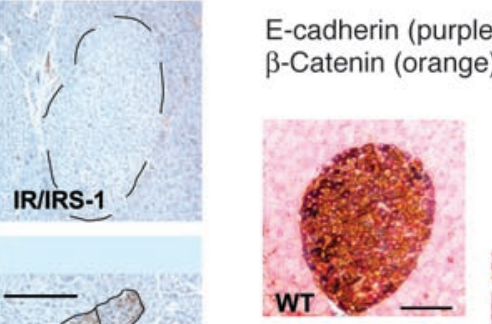
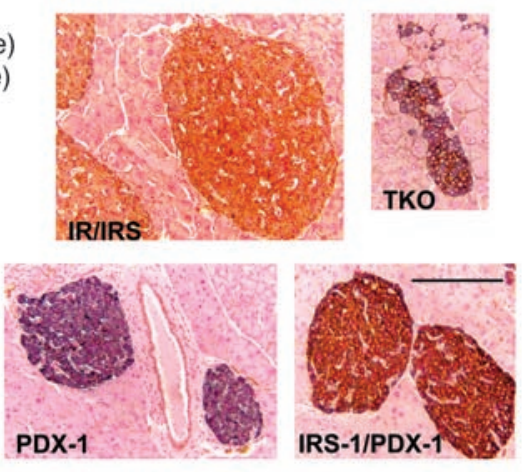

Figure 5

Immunostaining for pancreatic ductal marker, and markers for cell death and neogenesis. (A and B) Representative sections of pancreas from different genotypes, showing islets stained for PCNA (A) and caspase-3 (B). Note the magnified image for TKO. (C) Consecutive sections of pancreas from IR/IRS-1 mice immunostained for PCNA (left panels) or costained for insulin (green) and DBA-lectin immunohistochemistry for duct-specific glycoconjugates (red) (upper right panel). The lower right panels show 2 magnified images $(\times 60)$ of islet cells from IR/IRS-1 mice; the arrows point to cells positive for PCNA (purple chromogen) that also stain positive for nuclear $\beta$-catenin (blue chromogen). (D) Pancreas sections from WT, IR/IRS-1, PDX-1 heterozygous, IRS-1/PDX-1, and TKO mice costained for E-cadherin (purple) and $\beta$-catenin (orange) as described in Methods. Scale bars: $50 \mu \mathrm{m}$.

colocalization of PCNA and $\beta$-catenin in the nucleus strongly suggests the involvement of $\beta$-catenin in active proliferation (Figure $5 \mathrm{C}$, lower right panels). On the other hand, the morphological changes and downregulation of E-cadherin was not observed in TKO or IRS-1/PDX-1 islets, which suggests a limited ability of the mutant $\beta$ cells to respond to the insulin resistance in the presence of PDX-1 haploinsufficiency. Surprisingly, $\beta$ cells in TKO islets stained positive for caspase- 3 , suggesting a loss of $\beta$ cells due to apoptosis (Figure 5B).

PDX-1 haploinsufficiency limits islet byperplasia in a second model of insulin resistance, the LIRKO. In the next series of studies, we examined whether PDX-1 haploinsufficiency can also limit the islet hyperplastic response in other models of insulin resistance. We chose to examine the role of PDX-1 in the LIRKO mouse, which also shows severe hyperinsulinemia and robust islet hyperplasia (14). We generated compound KOs by crossing PDX-1 heterozygotes with mice bearing a homozygous deletion of exon 4 of the IR gene. This model of insulin resistance has been described earlier (14). Again, we observed an approximately 4-fold increase in $\beta$ cell mass in LIRKOs, while in contrast, in LIRKO/ PDX-1 compound KOs, the hyperplastic response was virtually absent and instead the islets were small with non- $\beta$ cells scattered throughout the islet (Figure 6, A and B). Thus, haploinsufficiency of PDX-1 limited the ability of islets to compensate in a second model, suggesting that the homeodomain protein is a crucial component in the $\beta$ cell proliferative response to insulin resistance. As in the IR/IRS-1 model, hyperplastic islets in the LIRKO mice showed alterations in the adherens junction (Figure 6C). Furthermore, the hyperplastic islets in LIRKOs also showed an absence of E-cadherin, pointing to a common pathway for $\beta$ cell expansion that critically requires the presence of
Table 1

Reduced number of PCNA+ cells and increased number of caspase- $3^{+}$cells in TKO islets

$\begin{array}{lccccccc} & \text { WT } & \text { IR } & \text { IRS-1 } & \text { PDX-1 } & \text { IR/IRS-1 } & \text { IRS-1/PDX-1 } & \text { TKO } \\ \text { PCNA }+ \text { cells per islet } & 0.5 & 0.3 & 0.3 & 0.6 & 28 & 4 & 0.1 \\ \text { Caspase-3+ cells per islet } & 1.3 & 2.2 & 1.8 & 3.1 & 4.1 & 11.2 & 26.4\end{array}$

$\mathrm{PCNA}^{+}$cells and caspase- $3^{+}$cells in islets in compound $\mathrm{KO}$ mice. At least 30 islets from 2 different mice were scored for each genotype. The average of data from at least 2 different animals in each group is shown. 


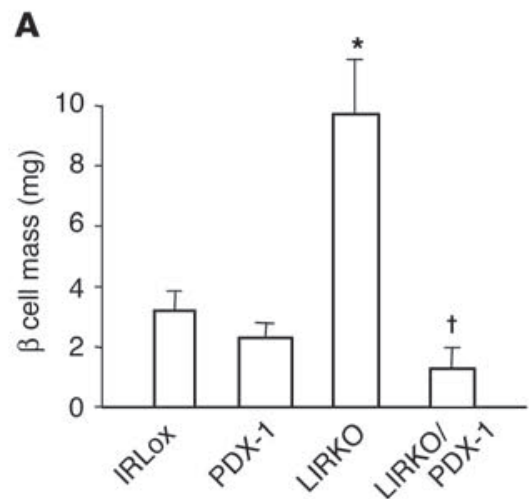

B Insulin (purple)

Glucagon (green)

Somatostatin (red)

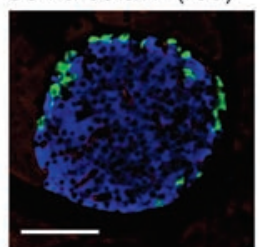

IRLox

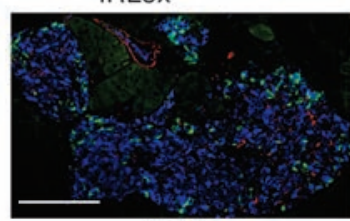

LIRKO

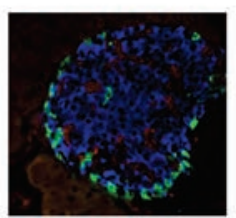

PDX-1

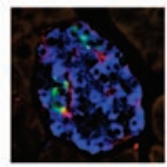

LIRKO/PDX-1
C
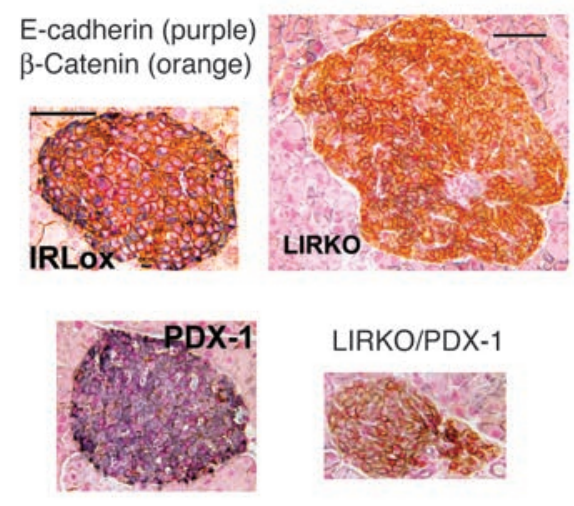

LIRKO/PDX-1

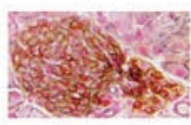

Figure 6

Alterations in a second model of islet hyperplasia, the LIRKO. (A) $\beta$ Cell mass was estimated by morphometric analysis as described in Methods. ${ }^{*} P<0.05$, LIRKO vs. IRLox (control), PDX-1, or LIRKO/PDX-1; ${ }^{\dagger} P<0.05$, LIRKO/PDX-1 vs. IRLox or PDX-1 ( $\left.n=4-6\right)$. (B) Representative islets from pancreas sections from the LIRKO group, costained for insulin (purple), glucagon (green), and somatostatin (red). (C) Representative pancreas sections from IRLox, PDX-1, LIRKO, and LIRKO/PDX-1 mice, costained for E-cadherin (purple) and $\beta$-catenin (orange) as described in Methods. Scale bars: $50 \mu \mathrm{m}$.

both copies of the PDX-1 gene. Consistent with earlier reports, we did not observe significant differences in non- $\beta$ cells among the groups (Figure 6B).

\section{Discussion}

In the present study, we have used 2 independent models of insulin resistance to demonstrate that PDX-1 is an important regulator of $\beta$ cell replication even in postdevelopmental states of $\beta$ cell growth. Indeed, PDX-1 is linked to $\beta$ cell neogenesis in the NOD mouse and in a model of pancreatic injury (partialpancreatectomy), and is associated with increased proliferation of isolated duct cells $(38,39)$. The lineage tracing of $\beta$ cells and the mechanism of replication of adult $\beta$ cells are currently areas of intense research. While ductal cells have been thought to be precursors of islet neogenesis, in the 2 models examined in this study, the proliferating $\mathrm{PCNA}^{+}$cells in the actively expanding islet are negative for a ductal marker. Furthermore, the fact that the associated changes in the composition of the adherens junction occurred only in large islets in the insulin-resistant mice indicates a coordinated and regulated process of $\beta$ cell replication. These observations are supported by several studies reporting the molecular mechanisms of other rapidly expanding epithelial cells, which show results remarkably similar to our own $(40,41)$.

Although the specific molecular mechanism(s) by which PDX-1 modulates $\beta$ cell replication is not fully understood, the low expression of $\beta$-catenin in $\beta$ cells in the PDX- 1 islet and the nuclear translocation of $\beta$-catenin in islet hyperplasia in the IR/IRS-1 mice suggest an association between PDX- 1 and $\beta$-catenin in the coordination of $\beta$ cell expansion. A recent study reporting that $\beta$ cells in adult mice are generated from preexisting $\beta$ cells (18) favors replication as the process most actively involved in the compensatory response to insulin resistance. The profound decrease in $\beta$ cell mass due to apoptosis in the TKO group was unexpected. Studies on differentiated neuronal cells (42) suggest that apoptosis is a potential default mechanism secondary to an abortive attempt at entering the cell cycle. Such a hypothesis is consistent with the increased caspase- 3 staining in TKO islets, and the $\beta$ cell death in these mice may indeed be secondary to a failure to expand. PDX-1 has been reported to regulate the expansion of pancreatic endocrine cells during development, and PDX-1 has been considered a regulator of $\beta$ cell-specific genes and proteins in the mature $\beta$ cell. These observations provide direct evidence, for the first time to our knowledge, that in addition to its role in $\beta$ cell-specific gene regulation, PDX-1 interacts with the insulin signaling system and that the homeodomain protein is a critical regulator of $\beta$ cell plasticity for the maintenance of glucose homeostasis.

\section{Methods}

Animals. IR/IRS-1 mice, PDX-1 heterozygotes, and LIRKOs were maintained on the original mixed background $(\mathrm{C} 57 \mathrm{BL} / 6 \times 129 \mathrm{~Sv} \times \mathrm{DBA} / 2)$ and bred at the Brandeis Animal Facility (Brandeis University, Waltham, Massachusetts, USA) on a 12-hour light/12-hour dark cycle with ad lib water and food (Mouse Diet 9F; PMI Nutrition International). A mixed mating scheme was adopted for breeding the two groups of mice. In the first group, both males and females carrying either the IR, IRS-1, or PDX-1 alleles were used. In the second group, males and females which were homozygous for loxP sites (IRLox) were mated with mice expressing Cre recombinase on an albumin promoter. The breeding generated several genotypes for the 2 studies. The genotypes of mice in the IR/IRS-1/ PDX-1 study included the WT, IR, IRS-1, IR/IRS-1, PDX-1, IRS-1/PDX-1, IR/PDX-1, and TKO groups. In the LIRKO/PDX-1 study, the genotypes included mice homozygous for loxP sites (IRLox), PDX-1 mice, LIRKO mice, and LIRKO/PDX-1 mice. Genotyping was performed by PCR analysis of genomic DNA obtained from tail snips (9). All procedures were approved by the Joslin Diabetes Center Institutional Animal Care and Use Committee and performed in accordance with its guidelines.

Blood glucose, serum insulin, and C-peptide levels and pancreatic insulin content. Overnight fasting (14 hours) and fed glucose levels were measured by a glucometer (Elite; Bayer Corp.) using tail vein blood. Serum insulin levels were assayed by ELISA using mouse insulin standards (Crys- 
tal Chem Inc.). C-peptide was measured by an RIA kit (Linco Research Inc.). Pancreatic insulin content was measured in acid-ethanol extracts of homogenized pancreas as described previously (9). Glucose and insulin tolerance tests and acute phase insulin release experiments were performed essentially as described previously (9).

$\beta$ Cell mass and immunohistochemistry. Mice were anesthetized, and pancreata were rapidly dissected, weighed, fixed in Bouin's or $4 \%$ paraformaldehyde solution, embedded in paraffin, sectioned, and stained as described below. $\beta$ Cell mass was estimated by morphometric analysis as described previously $(9,14)$. Five-micrometer sections of paraffinembedded pancreas were dewaxed using xylene, rehydrated through serial dilutions of ethyl alcohol, and subjected to antigen retrieval using $10 \mathrm{mM}$ citrate ( $\mathrm{pH}$ 6.1) or DAKO High $\mathrm{pH}$ antigen-retrieval solution (DAKO Corp.). The sections were washed and stained with the respective antibodies in staining buffer with $100 \mathrm{mM} \mathrm{NaCl}, 3 \%$ BSA, $1 \%$ Triton $\mathrm{X}-100$, and $50 \mathrm{mM} \mathrm{NaPO}_{4}$ (pH 7.4). Primary antibodies included guinea pig anti-insulin (Linco Research Inc.) or sheep anti-insulin (The Binding Site Ltd.), rabbit anti-somatostatin (DAKO), mouse anti-glucagon (Sigma-Aldrich), rabbit anti-Glut2 (a gift from B. Thorens, University of Lausanne, Lausanne, Switzerland), rabbit anti-synaptophysin (DAKO), mouse anti-PCNA (DAKO), $\beta$-catenin (BD Biosciences - Pharmingen), E-cadherin (Valeant Pharmaceuticals), rabbit anti-caspase-3, and Texas red-conjugated lectin (EY Laboratories Inc.). The secondary antibodies used included Cy 2 and Cy3 fluorescent conjugated dyes (Jackson ImmunoResearch Laboratories Inc.). Sections were viewed and photographed using a DeltaVision deconvolution microscope (Applied Precision LLC). For immunohistochemistry, sections were stained with primary antibodies (DAKO Corp.), followed by incubation with secondary antibodies (Envision Plus; DAKO Corp.). For color development, diaminobenzidine (DAB) chromogen (DAKO) was used and counterstained with Mayer's hematoxylin (diluted 1:1). Several chromogens were used for counterstaining as follows: for PCNA and caspase-3, we used DAB hematoxylin counterstaining; for PCNA and $\beta$-catenin, we used grey-black immunoprecipitates (Vector SG; Vector Laboratories Inc.) to visualize $\beta$-catenin and VIP substrate (red-purple color; Vector Laboratories Inc.) to visualize PCNA; for $\beta$-catenin and E-cadherin immunostaining, $\beta$-catenin was visualized with DAB (brown color) and E-cadherin with brown-black immunoprecipitates (Vector SG; Vector Laboratories Inc.); Nuclear Red (DAKO Corp.) was used for counterstaining of nuclei.
Western blotting. Whole pancreata were removed from mice and promptly homogenized using a polytron in $5 \mathrm{ml}$ of $1 \% \mathrm{SDS} / 6 \mathrm{M}$ urea lysis buffer containing protease and phosphatase inhibitors. Islets were homogenized using a hand-held homogenizer in the SDS-urea lysis buffer and processed similarly to whole pancreata. Equal proteins from samples (assessed by Micro BCA protocols; Pierce Biotechnology Inc.) were resolved by $10 \%$ SDS-PAGE, and the gel was transferred to nitrocellulose and probed for PDX-1 protein with anti-PDX-1 antibody (a gift from J. Habener, Massachusetts General Hospital, Boston, Massachusetts, USA) or anti-CREB antibodies using ECL (Amersham Biosciences).

Statistical analysis. Statistical analysis was performed by the Student's $t$ test or ANOVA as appropriate, and values were considered significant at $P<0.05$.

\section{Acknowledgments}

The authors thank Rohan Humphrey for helpful discussions, Doug Hanahan for critical comments on the manuscript, and Julie Marr for excellent secretarial assistance. R.N. Kulkarni is supported by a K08 NIH Clinician Scientist Award (DK 02885), and U.S. Jhala is supported by a Start-up Research Grant from the Hillblom Foundation. This study was supported by NIH R01 grant DK 67536 (to R.N. Kulkarni), by NIH grants to C.R. Kahn and M. Montminy, and in part by the Juvenile Diabetes Research Foundation Center for Islet Transplantation at Harvard Medical School (Project no. 13 to R.N. Kulkarni), by the Beta Cell Biology Consortium Pilot and Feasibility grant U19 DK 42502-VUMC (to R.N. Kulkarni), and by the Diabetes and Endocrinology Research Center grant P30 DK 36836 (Specialized Assay and Advanced Microscopy Cores). M. Montminy acknowledges support by the Larry Hillblom Foundation.

Received for publication April 9, 2004, and accepted in revised form August 3, 2004.

Address correspondence to: Rohit N. Kulkarni, Joslin Diabetes Center, Room 602, One Joslin Place, Boston, Massachusetts 02215 , USA. Phone: (617) 713-3460; Fax: (617) 713-3476; E-mail: Rohit. Kulkarni@joslin.harvard.edu.

Rohit N. Kulkarni and Ulupi S. Jhala contributed equally to this work.
1. Kulkarni, R.N., and Kahn, C.R. 2001. Genetic models of insulin resistance: alterations in $\beta$-cell biology. In Molecular basis of pancreas development and function. J.F. Habener and M. Hussain, editors. Kluwer Academic Publishers. New York, New York, USA. 299-323.

2. Melloul, D., Marshak, C., and Cerasi, E. 2002. Regulation of insulin gene transcription. Diabetologia. 45:309-326.

3. Cheatham, B., and Kahn, C.R. 1995. Insulin action and the insulin signaling network. Endocr. Rev. 16:117-142.

4. Potter, E., Bergwitz, C., and Brabant, G. 1999. The cadherin-catenin system: implications for growth and differentiation of endocrine tissues. Endocr. Rev. 20:207-239.

5. Dutta, S., et al. 2001. PDX:PBX complexes are required for normal proliferation of pancreatic cells during development. Proc. Natl. Acad. Sci. U. S. A 98:1065-1070.

6. Asahara, H., Dutta, S., Kao, H.Y., Evans, R.M., and Montminy, M. 1999. Pbx-Hox heterodimers recruit coactivator-corepressor complexes in an isoformspecific manner. Mol. Cell. Biol. 19:8219-8225.

7. Kim, S.K., et al. 2002. Pbx1 inactivation disrupts pancreas development and in Ipf1-deficient mice promotes diabetes mellitus. Nat. Genet. 30:430-435.

8. Johnson, J.D., et al. 2003. Increased islet apoptosis in $\mathrm{Pd} \times 1^{+/-}$mice. J. Clin. Invest. 111:1147-1160. doi:10.1172/JCI200316537.

9. Kulkarni, R.N., et al. 1999. Tissue-specific knockout of the insulin receptor in pancreatic $\beta$ cells creates an insulin secretory defect similar to that in Type 2 diabetes. Cell. 96:329-339.

10. Otani, K., et al. 2004. Reduced beta-cell mass and altered glucose sensing impair insulin-secretory function in betaIRKO mice. Am. J. Physiol. Endocrinol. Metab. 286:E41-E49.

11. Kulkarni, R.N., et al. 1999. Altered function of insulin receptor substrate-1-deficient mouse islets and cultured beta-cell lines. J. Clin. Invest. 104:R69-R75.

12. Kubota, N., et al. 2000. Disruption of insulin receptor substrate 2 causes type 2 diabetes because of liver insulin resistance and lack of compensatory $\beta$-cell hyperplasia. Diabetes. 49:1880-1889.

13. Bruning, J.C., et al. 1997. Development of a novel polygenic model of NIDDM in mice heterozygous for IR and IRS-1 null alleles. Cell. 88:561-572.

14. Michael, M.D., et al. 2000. Loss of insulin signaling in hepatocytes leads to severe insulin resistance and progressive hepatic dysfunction. Mol. Cell. 6:87-97.

15. Withers, D.J., et al. 1998. Disruption of IRS-2 causes type 2 diabetes in mice. Nature. 391:900-904.

16. Kitamura, T., et al. 2002. The forkhead transcription factor Foxo1 links insulin signaling to Pdx1 regulation of pancreatic $\beta$ cell growth. J. Clin. Invest. 110:1839-1847. doi:10.1172/JCI200216857.

17. Suzuki, R., et al. 2003. Pdx1 expression in Irs2-deficient mouse beta-cells is regulated in a strain-dependent manner. J. Biol. Chem. 278:43691-43698.

18. Dor, Y., Brown, J., Martinez, O.I., and Melton, D.A 2004. Adult pancreatic beta-cells are formed by self-duplication rather than stem-cell differentiation. Nature. 429:41-46.

19. Araki, E., et al. 1994. Alternative pathway of insulin signalling in mice with targeted disruption of the IRS-1 gene. Nature. 372:186-190.

20. Kulkarni, R.N. 2002. Receptors for insulin and insulin-like growth factor-1 and insulin receptor substrate- 1 mediate pathways that regulate islet function. Biochem. Soc. Trans. 30:317-322.

21. Kulkarni, R.N., et al. 2002. $\beta$-Cell-specific deletion of the Igf1 receptor leads to hyperinsulinemia and glucose intolerance but does not alter $\beta$-cell mass. Nat. Genet. 31:111-115.

22. Xuan, S., et al. 2002. Defective insulin secretion in pancreatic $\beta$ cells lacking type 1 IGF receptor. J. Clin. Invest. 110:1011-1019. doi:10.1172/ 
JCI200215276

23. Jonsson, J., Carlsson, L., Edlund, T., and Edlund, H. 1994. Insulin-promoter-factor 1 is required for pancreas development in mice. Nature. 371:606-609.

24. Waeber, G., Thompson, N., Nicod, P., and Bonny, C. 1996. Transcriptional activation of the GLUT2 gene by the IPF-1/STF-1/IDX-1 homeobox factor. Mol. Endocrinol. 10:1327-1334.

25. Leibiger, B., et al. 2001. Selective insulin signaling through A and B insulin receptors regulates transcription of insulin and glucokinase genes in pancreatic beta cells. Mol. Cell. 7:559-570.

26. Da Silva, X., Qian, Q., Cullen, P.J., and Rutter, G.A. 2004. Distinct roles for insulin and insulin-like growth factor-1 receptors in pancreatic beta-cell glucose sensing revealed by RNA silencing. Biochem. J. 377:149-158.

27. Shih, D.Q., et al. 2002. Profound defects in pancreatic beta-cell function in mice with combined heterozygous mutations in Pdx-1, Hnf-1alpha, and Hnf-3beta. Proc. Natl. Acad. Sci. U. S. A. 99:3818-3823.

28. Kulkarni, R.N., et al. 2003. Impact of genetic background on development of hyperinsulinemia and diabetes in insulin receptor/insulin receptor substrate-1 double heterozygous mice. Diabetes. 52:1528-1534

29. Kido, Y., et al. 2000. Tissue-specific insulin resistance in mice with mutations in the insulin receptor, IRS-1, and IRS-2. J. Clin. Invest. 105:199-205.

30. Dutta, S., Bonner-Weir, S., Montminy, M., and Wright, C. 1998. Regulatory factor linked to lateonset diabetes? Nature. 392:560.

31. Bonner-Weir, S. 2000. Perspective. Postnatal pancreatic beta cell growth. Endocrinology. 141:1926-1929.

32. Kelman, Z. 1997. PCNA: structure, functions and interactions. Oncogene. 14:629-640.

33. Budihardjo, I., Oliver, H., Lutter, M., Luo, X., and Wang, X. 1999. Biochemical pathways of caspase activation during apoptosis. Annu. Rev. Cell Dev. Biol. 15:269-290.

34. Kobayashi, H., et al. 2002. Lectin as a marker for staining and purification of embryonic pancreatic epithelium. Biochem. Biophys. Res. Commun. 293:691-697.

35. Dahl, U., Sjodin, A., and Semb, H. 1996. Cadherins regulate aggregation of pancreatic beta-cells in vivo. Development. 122:2895-2902.

36. Williams, E.J., Furness, J., Walsh, F.S., and Doherty, P. 1994. Activation of the FGF receptor underlies neurite outgrowth stimulated by L1, N-CAM, and $\mathrm{N}$-cadherin. Neuron. 13:583-594.

37. Lopez, T., and Hanahan, D. 2002. Elevated levels of IGF-1 receptor convey invasive and metastatic capability in a mouse model of pancreatic islet tumorigenesis. Cancer Cell. 1:339-353.

38. O'Reilly, L.A., et al. 1997. $\alpha$-Cell neogenesis in an animal model of IDDM. Diabetes. 46:599-606.

39. Sharma, A., et al. 1999. The homeodomain protein IDX-1 increases after an early burst of proliferation during pancreatic regeneration. Diabetes. 48:507-513.

40. Savagner, P. 2001. Leaving the neighborhood: molecular mechanisms involved during epithelialmesenchymal transition. Bioessays. 23:912-923.

41. Thiery, J.P. 2002. Epithelial-mesenchymal transitions in tumour progression. Nat. Rev. Cancer. 2:442-454.

42. Becker, E.B., and Bonni, A. 2004. Cell cycle regulation of neuronal apoptosis in development and disease. Prog. Neurobiol. 72:1-25. 